\title{
Initiative Study for High Cholesterol Inhibitor with Various Components from Natural Herb Medicine
}

\author{
Tai Jin Kim ${ }^{1 *}$, Myung Rae Park ${ }^{1}$, Jong Woog Lee ${ }^{2}$, Yong Chul Hur ${ }^{3}$ and In Sop Shim ${ }^{4}$ \\ ${ }^{1}$ Department of Chemical Engineering, University of Suwon, Hwasung City, Republic of Korea \\ ${ }^{2}$ Daewoong Pharmaceutical, Gangnam-gu, Seoul, Republic of Korea \\ ${ }^{3}$ Kolmar Korea Co., Ltd., Sejong, Republic of Korea
}

${ }^{4}$ Medical School, Kyung Hee University, Seoul, Republic of Korea

*Corresponding author: Tai Jin Kim, Department of Chemical Engineering, University of Suwon, Hwasung City, Republic of Korea

\section{ARTICLE INFO}

Received: 幽 July 30, 2019

Published: 幽 August 13, 2019

\section{ABSTRACT}

Keywords: Initiative; High Cholesterol Inhibitor; Natural; Herb; Medicine

Citation: Tai Jin Kim, Myung Rae Park, Jong Woog Lee, Yong Chul Hur, In Sop Shim. Initiative Study for High Cholesterol Inhibitor with Various Components from Natural Herb Medicine. Biomed J Sci \& Tech Res 20(3)-2019. BJSTR. MS.ID.003468.

\section{Introduction}

$40 \%$ of medicines were induced from natural resources while one candidate could be available from 5,000 organic syntheses. It is therefore important to screen the effective materials from natural medicine to ultimately scale up the effective components by either chemical or biotechnological synthesis. The purpose of the present study is to analyze several candidates of natural herbs and to examine their efficacy in spontaneously hypertensive rats as a high cholesterol inhibitor.

\section{Experiment}

9 natural herbs (ginseng, ganoderma, iucidum, coriolus versicolor, angelica gigas nakai, epimedium Koreanum nakai, eucommia ulmoides, Lycium chinense Miller, cassiae semen, pine needle) were boiled 4 hours to be centrifuged at 10,000 rpm for $20 \mathrm{~min}$. Their supernatants were under rotary evaporator $\left(73^{\circ} \mathrm{C}\right.$, $1.15 \mathrm{rps}, 1 \mathrm{hr} 10 \mathrm{~min}) .5 \mathrm{~g}$ activated carbon was added at $30^{\circ} \mathrm{C}$, which was shake and filtered by vacuum filtration. Animal experiments were carried out with 24 Spontaneously Hypertensive Rats (SHR) for 3 months. Blood samples were analyzed for total cholesterol, triglycerides, HDL cholesterol and total lipid. Blood pressure was measured by tail cuff method with a PB Recorder 8006 (Ugo Basile). There were 4 groups for animal tests : Group I (Distilled water), Group II (Natural herbs extract), Group III (Sodium hyaluronate) and Group IV (Sodium hyaluronate + Natural herbs extract).

\section{Results}

FT-IR spectrum for 9 natural herbs and 1 standard were shown in Figure 1.
a) Ginseng,
b) Ganoderma Iucidum,
c) Coriolus Versicolor,
d) Angelica Gigas Nakai,
e) Epimedium Koreanum Nakai,
f) Eucommia Ulmoides,
g) Lycium Chinense Miller,
h) Cassiae Semen, 


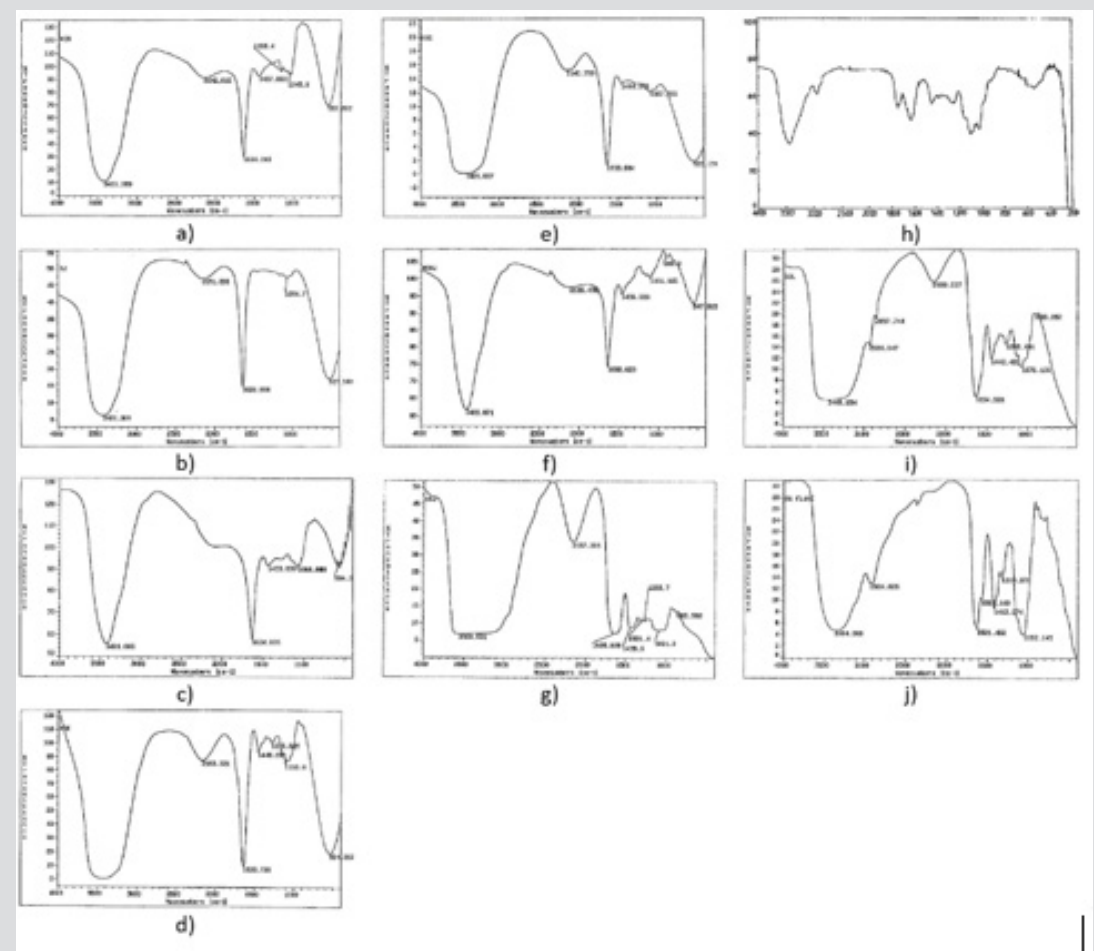

Figure 1: FT-IR-Spectrum of 9 Natural Herbs and 1 Standard.

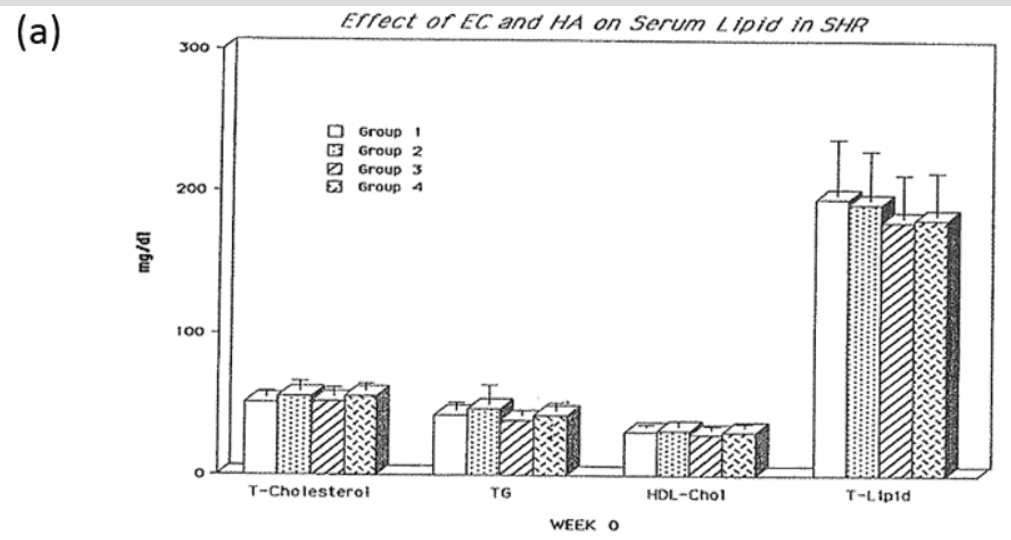

(b)

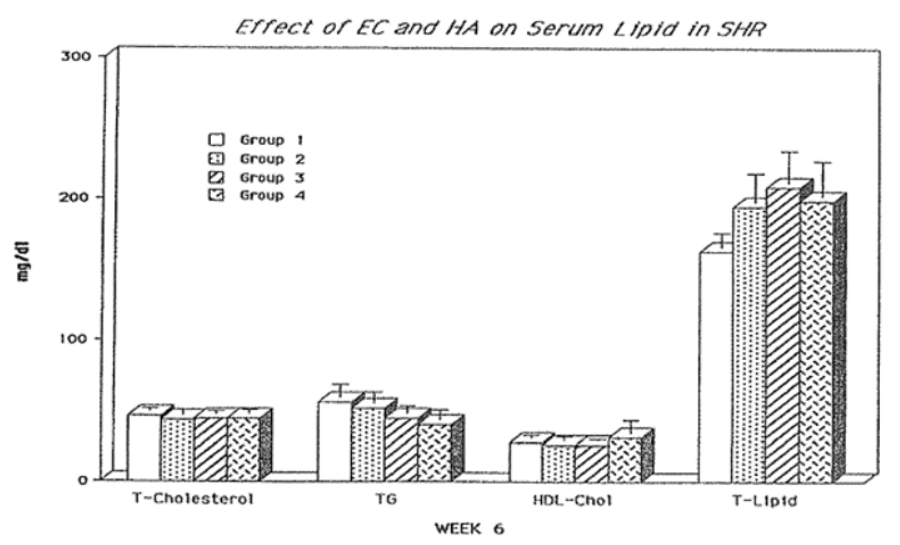

Figure 2: Biochemical Compositions of SHR Blood of Individual Group.

(a) Before Drug Administration.

(b) After Six Weeks of Drug Administration. 


\section{Conclusion}

1) An initiative study by FT-IR scanning proposed that eucommia ulmoides, lycium chinense miller and pine needle showed profiles close to that of sodium hyaluronate biopolymer.

2) 24 spontaneously hypertensive rats were fed for 130 days and bloods from their veins were sampled every 10 days. The group fed by a mixture of extracts from eucommia ulmoides,

ISSN: 2574-1241

DOI: $10.26717 / B J S T R .2019 .20 .003468$

Tai Jin Kim. Biomed J Sci \& Tech Res

This work is licensed under Creative

Commons Attribution 4.0 License

Submission Link: https://biomedres.us/submit-manuscript.php lycium chinense miller and pine needle with sodium hyaluronate biopolymer (Group IV), showed decreases in cholesterol (15\%) and total lipid (22\%) while the ratio of HDL cholesterol with respect to total cholesterol was increased.

3) It is therefore suggested that a mixture of extracts from eucommia ulmoides, lycium chinense miller and pine needle with sodium hyaluronate biopolymer can be a good candidate for a high cholesterol inhibitor.

$\begin{array}{ll}\text { BIOMEDICAL } & \text { Assets of Publishing with us } \\ \text { RESEARCHES } & \text { - Global archiving of articles } \\ & \text { - Immediate, unrestricted online access } \\ & \text { - Rigorous Peer Review Process } \\ \end{array}$

